\title{
INTERSECTION DETECTION BASED ON QUALITATIVE SPATIAL REASONING ON STOPPING POINT CLUSTERS
}

\author{
S. Zourlidou *, M. Sester \\ Institute of Cartography and Geoinformatics, Leibniz University, Appelstraße 9a, 30167, Hannover, Germany - \\ (zourlidou, sester)@ikg.uni-hannover.de
}

Commission II, WG II/3

KEY WORDS: clustering, spatial reasoning, relational reasoning, qualitative cluster reasoning, geospatial analysis, point data analysis, intersection detection, rule-sensing, semantic trajectories, stops and moves

\begin{abstract}
:
The purpose of this research is to propose and test a method for detecting intersections by analysing collectively acquired trajectories of moving vehicles. Instead of solely relying on the geometric features of the trajectories, such as heading changes, which may indicate turning points and consequently intersections, we extract semantic features of the trajectories in form of sequences of stops and moves. Under this spatiotemporal prism, the extracted semantic information which indicates where vehicles stop can reveal important locations, such as junctions. The advantage of the proposed approach in comparison with existing turning-points oriented approaches is that it can detect intersections even when not all the crossing road segments are sampled and therefore no turning points are observed in the trajectories. The challenge with this approach is that first of all, not all vehicles stop at the same location thus, the stop-location is blurred along the direction of the road; this, secondly, leads to the effect that nearby junctions can induce similar stop-locations. As a first step, a density-based clustering is applied on the layer of stop observations and clusters of stop events are found. Representative points of the clusters are determined (one per cluster) and in a last step the existence of an intersection is clarified based on spatial relational cluster reasoning, with which less informative geospatial clusters, in terms of whether a junction exists and where its centre lies, are transformed in more informative ones. Relational reasoning criteria, based on the relative orientation of the clusters with their adjacent ones are discussed for making sense of the relation that connects them, and finally for forming groups of stop events that belong to the same junction.
\end{abstract}

\section{INTRODUCTION}

The essential human need for accessing goods and services as well as for fulfilling other personal, social and professional needs makes mobility an important aspect of everyone's daily life. No doubt GPS technologies have played an important role of how the mobility and transport needs are materialized and given that maps are the core components of such systems, the requirement of up-to-date and detailed maps is regarded of high priority for both mapping providers and LBS (location-based services) users.

An up-to-date map reflects the real topological and topographical features of the road network, a task which turns out to be very challenging. Among the other consequences of the increased mobility, one can enlist the continuous evolution of the road network (new road segments, topological changes, temporal closures, etc.) and other features that are mapped onto the maps which also change over time (e.g. points of interest, speed limits, etc). When maps fail to fulfill the prerequisite of being up to date, then the task of navigation or that of other LBSs, that aim to assist users, fails as well, if not saying that it additionally complicates the reaching to the destination in the first case and leaves users dissatisfied with the offered service in the second one. According to Mapscape (2016), roads change by as much as $15 \%$ a year, a fact that further highlights the importance of the map update process. Surveying for mapping is a time and cost expensive procedure which sets limitations on the update potential. Hence, profit and nonprofit map institutions aim at overcoming these restrictions by using crowdsourced GPS tracks recorded by GPS receivers or user generated content (UGC), enabling that way dynamically selfupdated maps and mass-market mapping (e.g. OpenStreetMap). The research interest in that case focuses mainly on the extraction of the geometrical and topological features of the road network. A wide range of different approaches have been proposed for automating the map construction process (Cao and Krumm, 2009; Karagiorgou and Pfoser, 2012; Biagioni and Eriksson, 2012; Wang, et al 2013) and for improving the existing road data by harnessing incoming new information from GPS traces (Zhang and Sester, 2010; Zhang et al, 2010). Similar approaches go beyond the inference of the geometry and connectivity of the roads and extract also lane features number of lanes, lane splits and merges- (Schroedl et al, 2004; Chen and Krumm, 2010), context information in the form of interesting activities and places (Agamennoni, 2009), road features such as road class and road name (Li, 2015) and average and maximum velocity per road segment (Niehofer et $a l, 2009)$. Furthermore, with the recent advance of embedded sensing devices (e.g. GPS devices, accelerometers in smartphones), Pervasive Urban Sensing (PUS) has become increasingly popular for applications that vary from real-time traffic light sensing (Zhu et al, 2013) and parking spot occupancy estimations (Mathur et al, 2010) based on probe vehicle data, to environmental noise monitoring (Mohan et al, 2008) and road and traffic condition sensing (bumps, potholes, hard braking, honking) based either on mobile devices (Mohan et al, 2008) or on sensor-equipped vehicles (Eriksson, 2008).

* Corresponding author 
This paper is motivated by authors' on-going research on road regulation sensing (Zourlidou and Sester, 2015b; Sester, et al, 2015) and regulation-aware navigation (Zourlidou and Sester, 2015a) and aims to explore the possibility of detecting intersections using stop event locations that are logged through vehicles' CAN-Bus. As it is explained later, processing data from multiple cars in an incremental way can reveal such information that could be difficult to be recovered with existing methods. By achieving it, digital maps can be dynamically enriched with accurate and up-to-date semantics.

The remainder of this paper is organized as follows: in subsection 2.1 the motivation of this research is discussed followed by 2.2 where methods for detecting intersections are reviewed. It is shown that given nearly all of them are based on clustering turning points, there is a need of inventing a different approach for the case where not all crossing road segments are sampled and therefore no turning points are observed in the dataset. Section 3 discusses the idea of clustering stop events, highlighting the challenges through examples where existing clustering methods were applied and shown not to properly work in the context of the problem we examine here (subsection 3.2). Subsection 3.3 presents a new method for discovering intersections based on density-based clustering coupled with some qualitative checks of nearby clusters that advocate merging of the latter under conditions. Conclusions and suggestions drawn from the findings are reported in Section 4.

\section{INTERSECTION DETECTION: MOTIVATION AND EXISTING METHODS BASED}

\subsection{Motivation}

As mentioned earlier in section 1, the motivation for detecting intersections originates from authors' research interest in enhancing maps with traffic controls. By traffic controls we mean traffic rules such as traffic signs or others that are due to physical restrictions that modern urban design introduces for improving traffic behavior (Hamilton-Baillie and Jones, 2005). Instead of mining such traffic controls using data from vehicles that are equipped with special cameras (e.g. stereo cameras) and by applying computer-vision methods (Fairfield and Urmson, 2011; Chigorin and Konushin, 2013), we currently explore ways of extracting such information relying on in-car sensors such as GPS receivers, blinker and brake signals (Zourlidou and Sester, 2015a; 2015b). A relevant problem in the context of inferring traffic controls is that of junction classification according to their type of control (we distinguish four types of intersection control: stop-, traffic signal-, yield-, unrestrictedcontrolled). In other words, we want to infer the type of intersection control given data acquired from in-vehicle sensors, without the position of intersections being given. The requirement of solving this classification problem without knowing the positions of intersections leads naturally to the need of first identifying these positions

One of the main aspects that differentiates our proposed approach from existing ones is that it doesn't presuppose the intersection positions. For example, Pribe and Rogers (1999) describe a method for learning to associate driver behavior with a subset of traffic controls (stoplights and stop signs) regarding though the positions of the intersections as known. Similarly, $\mathrm{Hu}$ et al (2013) compare two different methods for crowedsourced traffic regulator detection and consider the map as given. As discussed in Section 1, the road network undergoes daily interventions which means that current intersections may be reformed or new ones be created, a fact which emphasizes the need for intersection detection in an automatic and dynamic way. Having said that and before we present our proposed method which is independent from turning points (see paragraph 2.3), in the next section we make a short review of methods that serve the same purpose, underscoring their weakness to be applicable in datasets with non fully sampled intersections (samples are not available from all road segments that participate in intersections' composition).

\subsection{Existing methods for intersection detection}

Methods for detecting intersections can be categorized in two main categories: raster- and point-based where by point we mean a GPS trace. Since our dataset is composed of samples of the second category, our review is focusing here on known point-based methods.

Makris and Ellis (2002, 2003) propose an activity-based semantic scene modelling method for deriving the structure of scenes (spatial representation). Trajectories are obtained by tracking moving objects (pedestrians) and being processed for deriving semantic entities of the scene: entry/exit zones, routes, paths and junctions. As junctions are considered regions where routes cross each other. First models are learnt from trajectories by applying a geometrical analysis which compares the distance between a trajectory and an evolving route. At a second step paths are extracted from the routes by detecting route cross over points (Makris and Ellis, 2002, pp.897) that afterwards are classified as junctions. Combining these findings with other extracted semantic entities such entry/exit locations, the topological representation of the scene map is finally obtained.

Fathi and Krumm (2010) describe a method for detecting road intersections from GPS traces acquired from regular vehicles as a first step for road network construction. A localized shape descriptor is used to represent the local distribution and direction of the traces around a point and a classifier is trained using the Adaboost algorithm for learning to discriminate intersections from non-intersections. For ensuring the accurate localization of intersections' positions, the iterative closest point algorithm is applied as a final refining step of the resulted positions. A 64-bin descriptor (4 circles, 16 angular slices) was trained and tested and found to be able to distinguish between positive (intersections) and negative (non-intersections) feature vectors. Negative samples were shown to have a peak every 180 degrees (every 8 bins) which is justified by the fact that negative samples come usually from straight roads. Positive samples (three-, four-way intersections) were found to have a peak every 90 degrees (every 4 bins).

Karagiorgou and Pfoser (2012) propose an automatic road network generation algorithm using as input GPS tracks from a school bus fleet. The basic idea is first to identify turns and then find clusters of turns that are regarded as intersection nodes. Then the road network is generated by connecting intersection nodes with links derived from trajectories that exhibit turns at these intersections (pp. 93). For identifying turns a speed threshold of $40 \mathrm{Km} / \mathrm{h}$ was used as well as a direction threshold of $15^{\circ}$. Both thresholds were defined experimentally. The intuition behind the selection of these two measures as turn indicators, as authors explain, is that when a vehicle turns it reduces its speed and changes its direction.

Wu et al (2013) describe the intersections as transport hubs and propose a method for detecting them in order afterwards to use 
them for building and updating maps by harnessing coarsegained GPS traces. Similarly to Karagiorgou and Pfoser method, turning points are recognised (heading change greater than $45^{\circ}$ ) and used for defining converging points (see pp. 2960 for the definition). The position of intersections then is estimated by the centres of the clusters of the converging points applying a variant of the $\mathrm{X}$-means algorithm.

Last, Xie et al (2015) consider intersections as locations where users can change directions in multiple ways, regardless of the number of road segments that meet at a particular junction. The authors explain that junctions differentiate from bends due to the fact that in the latter case drivers are observed to change their direction only in a single way. So, turning points having previously be distinguished as not belonging to road bends are again employed for extracting intersections. A region-growinglike clustering technique based on the Euclidean distance between the turning points is proposed for grouping sequences of turning points into intersections. Clusters that contain few turning points are discarded at a post-processing step.

One can observe that all of these methods but the first one (Makris and Ellis, 2002) count on the common movement behaviour that vehicles show when crossing intersections: a significant change in their heading. Turning points are defined according to this condition and are clustered with different methods for finding their centres which represent intersection positions, whereas in Makris and Ellis (2002) the assumption is that a junction can be detected when two or more paths intersect each other. No doubt, these assumptions are plausible but by definition intersections which are represented in the dataset by trajectories that do not meet these preconditions are excluded from being detected (Figure 2, upper left and right). This means that in the case of either a non rich dataset or of one where less popular roads cross popular ones and given that vehicles tend to follow the latter, not all intersections can be derived by following one of these approaches, because samples from crossing trajectories or turning points would not be present (or would not be enough as in Figure 2, lower-left) in the dataset for all sampled junctions as opposed to Figure 2 lower right. Exactly this observation motivated this research on exploring alternative ways of detecting intersections and which is the topic of discussion of the next paragraph.
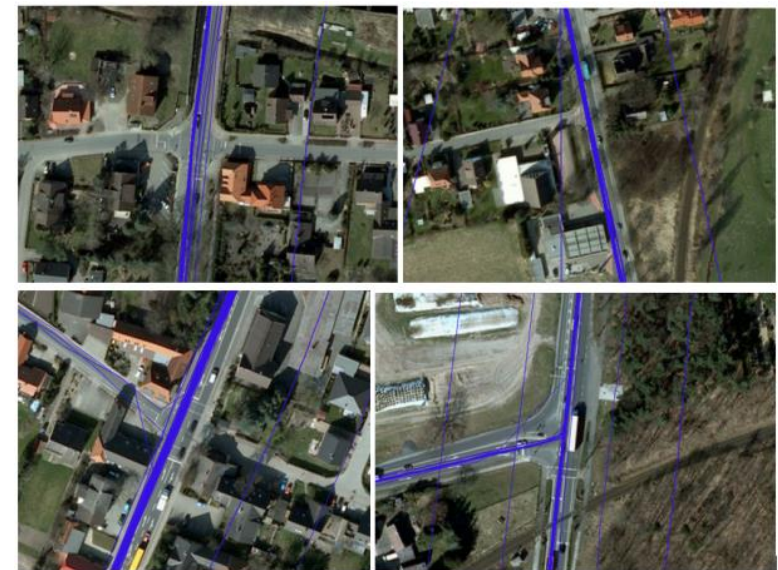

Figure 2. Upper-left: Intersection where no samples (trajectories) exist from all participating road segments that compose the intersection. Upper-right: T-junction where no samples (trajectories) exist from the secondary participating to

the junction road. Lower-left: Traffic light-controlled Tjunction, where only three samples exist in the dataset that turn at it. In contrast many samples cross straight over it. Lowerright: Example of a well- sampled intersection

\section{INTERSECTION DETECTION BASED ON STOP EVENTS CLUSTERS}

\subsection{Approach for stop events usage for junction discovery}

Generally speaking a trajectory represents the consecutive positions of a moving object over time. During their movement, moving objects change location, heading, speed, velocity and/or curvature, to mention some aspects of movement. Because normally these aspects are not changing continually over time, there are segments along the trajectories where these characteristics remain unchanged. Segmenting trajectories according to spatiotemporal criteria (e.g. Buchin et al, 2010) can reveal meaningful locations and behaviour of the moving object(s) along the trajectories (Sester et al, 2012).

In the context of the problem we examine here, we are interested in detecting intersections based on stop event locations, so we segment the trajectories in sequences of stop and moves. Such a segmentation has also been used for discovering interesting places in single trajectories (Palma et al, 2008) and for enriching trajectories with semantic geographical information (Alvares et al, 2007). Our scope is to find patterns of vehicles' movement and more specifically to detect reference spots $^{l}$ (Li et al, 2010,) by observing where vehicles stop, so the extraction of the "interesting places" here is defined by massively observing the same behaviour at the same location as opposed to Palma et al (2008) where interesting locations have a more "personalised" character².

The assumption for the usage of stop events to detect intersections is that vehicles stop more often at these locations (traffic lights, stop/yield signs,) than at others, so we assume that in intersection locations clusters of stop events can be observed. Figure 3 shows three intersections that correspond to this assumption with point symbols symbolizing positions where vehicles have stopped. Under this assumption,
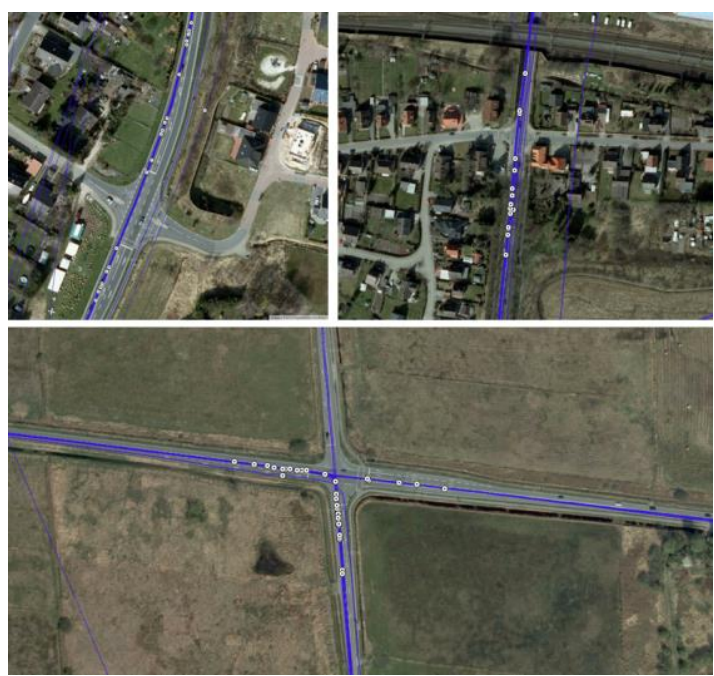

Figure 3. Stop events (white spheres) at controlled intersections

${ }^{1}$ Here the term reference spot denotes locations associated with a specific behaviour similarly to the original usage of the term that refers to locations associated with periodic behaviours.

${ }^{2}$ Interesting locations are mined by observing the moving behaviour of a single object. 


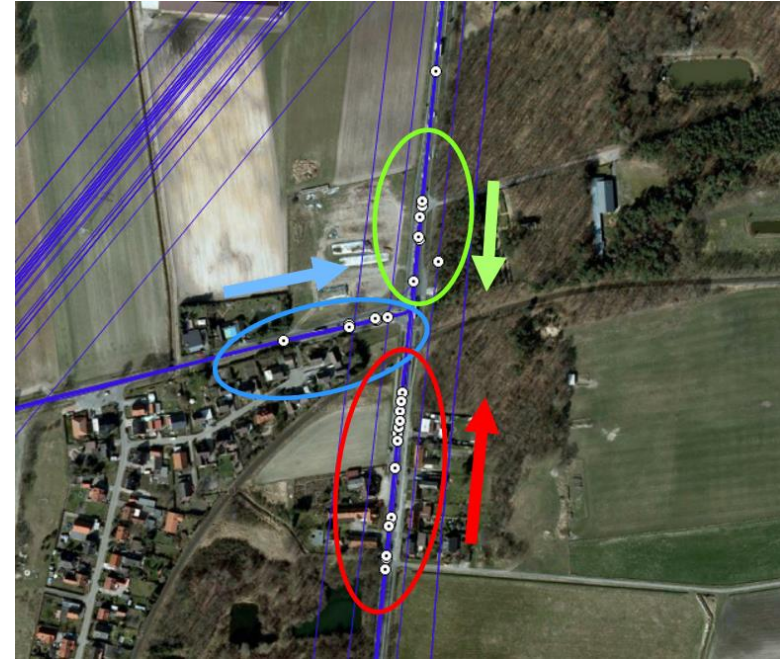

Figure 4. Stop events at a controlled T-junction. The idea for discovering intersections is based on the assumption that the centers of groups of nearby clusters of stop events from vehicles that moves in conflicting headings can reveal the position of the intersection

intersection locations can be detected by finding nearby clusters of stop events from vehicles that are moving in "conflicting" directions. Here "conflicting" has not necessary the meaning of an $180^{\circ}$ difference in heading from a referent heading (Figure 4 , green and red do have such difference in their heading). It more means a direction which differs greatly from the referent. For example in Figure 4, green and blue clusters differ in heading by more than 90 degrees and similarly do blue and red clusters. Therefore the aim is to find clusters of stop events from objects that are moving in conflicting headings and to estimate the centres of the intersections as those points where clusters' headings are intersecting. In the following paragraph the challenges of implementing this approach with known clustering techniques are discussed.

\subsection{Clustering stop events: challenges}

Three methods for clustering stop events were applied in our dataset:

1. Density-based clustering (DBSCAN, Ester et al, 1996) having included in the feature vector the position of stop events and the heading of vehicles' movement prior stance.

2. K-means classification followed by DBSCAN clustering:

a. K-means classification of stop events based on the heading of movement prior stance (16 classes).

b. DBSCAN clustering on the categories found in a. based on the position of stop events.

3. Kernel density estimation of the stop events.

As shown in Figure 5, the first two methods seemed to work generally well in locations where no (sampled) intersections are found close to each other or no random stops are observed after crossing the intersections. The problem of poor clustering occurred in scenaria as that shown in Figure 6a. Stop events that represent random stops after crossing intersections or stance at a near intersection were clustered erroneously in the same group, forming clusters that do not represent possible reference spots of common stop behaviour just before an intersection. In Figure $6 \mathrm{~b}$, the pink cluster contains samples that spread over two close junctions. An explanation is that random stop events between close intersections function as a "link" of the densities of their
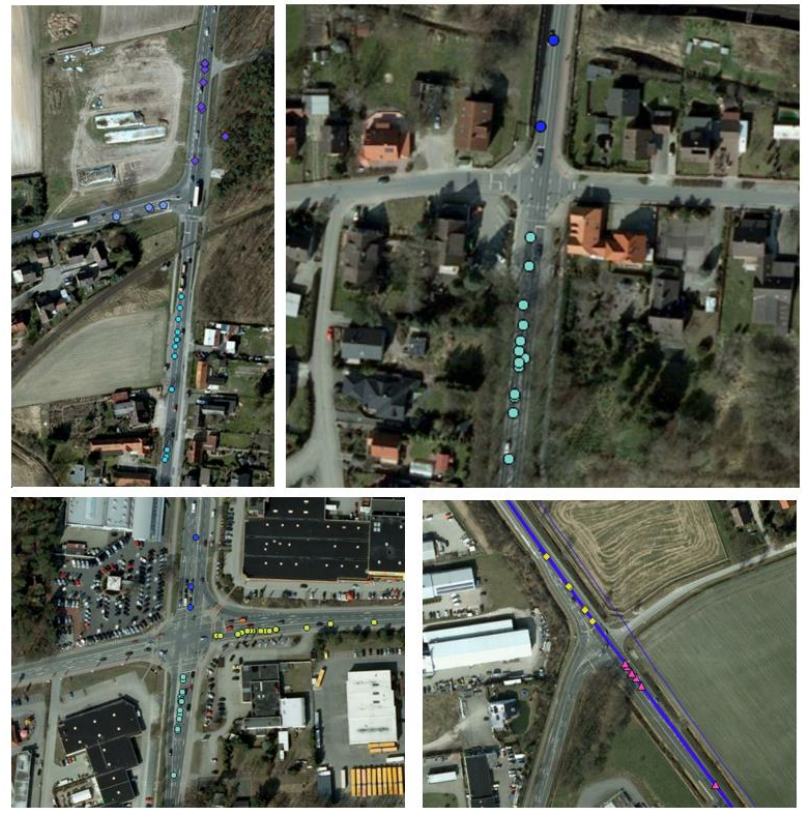

Figure 5. Clusters of stop events detected with methods 1 and 2

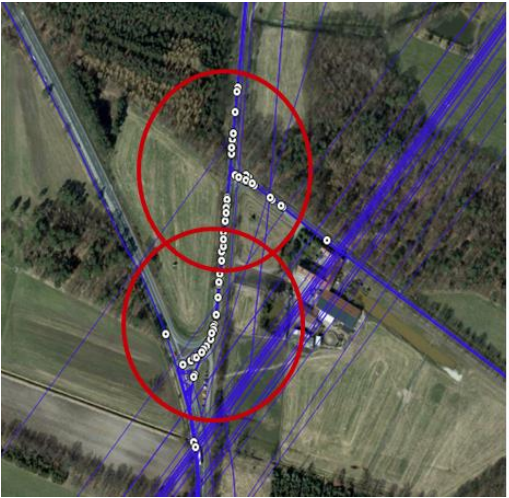

(a)

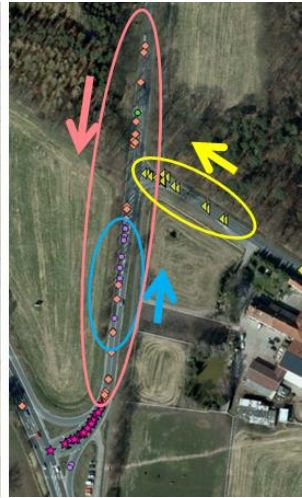

(b)
Figure 6. Clusters of stop events as detected with methods 1, 2
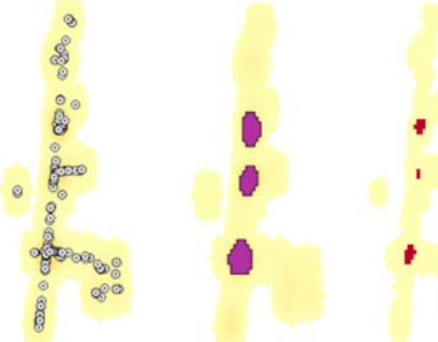

(a)

(b)
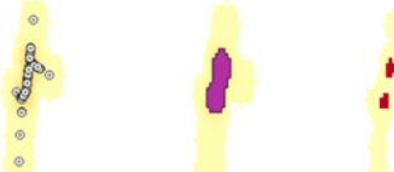

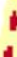

(c)

(d)

Figure 7. Clustering with kernel estimation. In (c) a low threshold has been used resulting in identifying two near intersections as one (see low part of the figures). In (d) a higher threshold exclude the in between the two high densities noisy samples and make them distinguishable 
stops events, linking them and finally distinguishing the two intersections as a single one. This motivated us to use the $3^{\text {rd }}$ method and try to exclude the noisy stop samples and find clusters (Kernel density estimation) with dense samples as shown in Figure 7. The problem with this method is that when a low threshold is set, near interactions are detected as being one (Figure 7c) and when increasing the threshold, near ones become distinctive (Figure 7d) but other intersections with low dense samples and previously distinctive are now excluded from being identified due to the high threshold. To overcome these shortcomings, a new method was designed that is introduced in the next subsection.

\subsection{Discovering intersections with stop events}

3.3.1 Method: A 3-step method is proposed for discovering intersections. The underlying idea is that the sought-after junction is close to the cluster end, namely the most distant point among cluster members along the direction of their movement (see Figure 8). Thus, first the clusters are detected, followed by an analysis of their heading and an identification of the cluster end closest to the junction.

The process is as follows:

1. Density-based clustering of the stop events including only the position in the feature vector (including also the heading and having normalised the feature vector, samples with a slight difference in heading with other close samples were remained unclassified).

2. Splitting clusters found at step 1 according to the heading of the movement of the members of the clusters before stance.

- Determination of representative points of the clusters (one per each).

3. Merging adjoining clusters by Region-growing clustering of the clusters found at step 2 using spatial reasoning criteria.

i. Random selection of a cluster. Then finding its nearby clusters. Merging adjacent clusters of same direction (representatives are recalculated).

ii. Clusters analysis using qualitative spatial reasoning. The algorithm terminates when all clusters have been analysed.

Generally speaking, qualitative spatial reasoning explains why clusters are there as opposed to (quantitative) clustering analysis that answers to what and where point data are aggregated. Including a rich set of qualitative spatial information, various qualitative spatial relations can be extracted from the data in terms of topology, distance and orientation, revealing novel patterns (Qu et al, 2010). In Figure 9 a hypothetical scenario of sampling a crossroad intersection and finding groups of near clusters is given. Nearby clusters of similar direction are merged and those having conflicting directions (such as in Figure 4 and 5) are classified in a new cluster, as they indicate a stop at the same intersection. This new cluster represents a group of clusters of stop events from vehicles that stopped in the same intersection and which were moving along different directions. As it can be seen in Figure 9, the relations of clusters and not the clusters per se inform us about the existence of an intersection. More specifically, the relative position of the clusters with each other is important for making sense of the total observed behaviour on the reference spot, and based on the spatial analysis of this behaviour the location can be characterized as intersection or not. Under this view, the clusters per se can be seen as indicators of (possible) reference spots, but it is the presence (and the direction) of other nearby clusters that explains or emphasizes why traffic participants behave similarly at that location and it consequently enable is of using these relations for reasoning about the type of location.

The spatial relations shown in the Figure 9 are exemplary. Here clusters are located in $90^{\circ}$ or $180^{\circ}$ degrees relative to neighbour ones. The same idea nevertheless applies also to other types of junctions (e.g. Y-, T-junctions). It is only needed to define how big the difference between the directions of the samples can be to be considered as "conflicting"( See subsection 3.1) with each other.

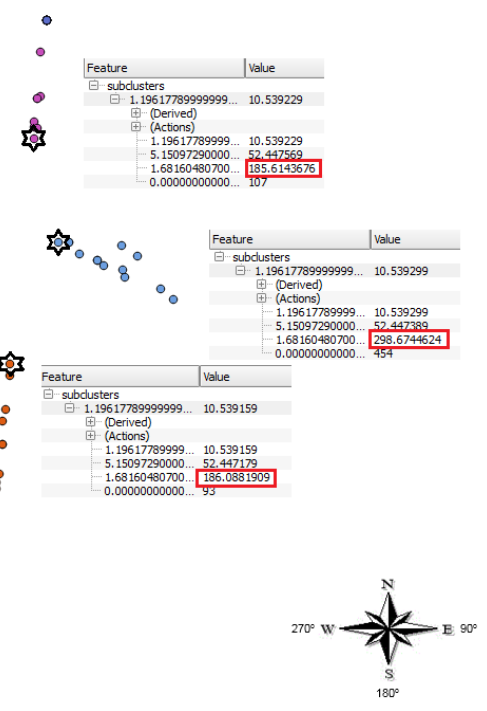

Figure 8. Representatives of the clusters are symbolized with six-pointed stars. On their right are given their coordinates and the heading of their movement (red rectangle)

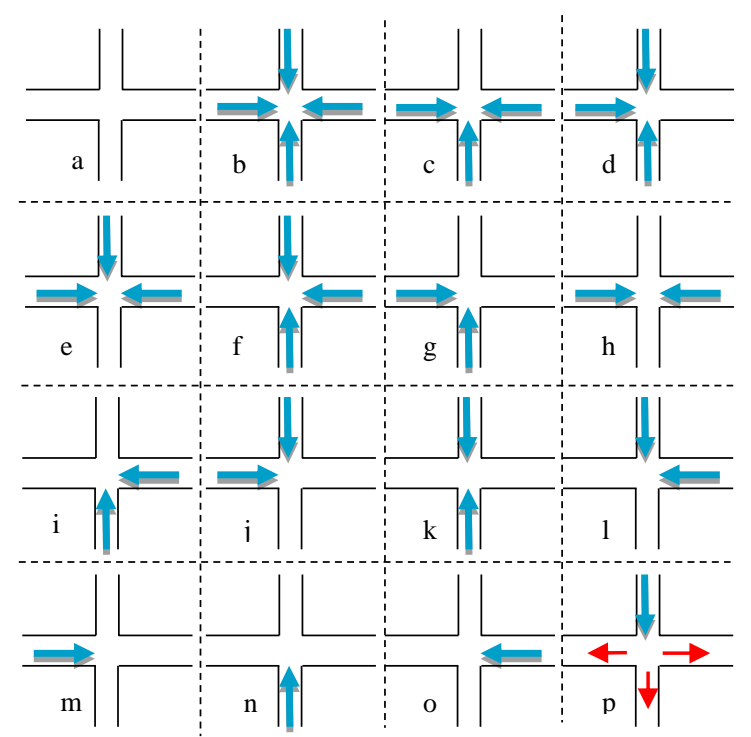

Figure 9. Blue arrows symbolize clusters of stop events which come from vehicles moving along directions indicated by the arrows. They are considered as valid combinations of clusters

that contribute to junction discovery. These clusters are discovered at step 1 of the method described in this subsection.

From (b)-(l), groups of four (a), three (c-f) and two (g-l) clusters, that have conflicting headings suggest the probable existence of an intersection. On all road segments, it's still possible, clusters from noisy stop events to be found ((p), red arrows), but since they do not express "conflict" with the other cluster(s) observed in the same location (blue cluster in (p)), their representation was omitted for clarity reasons 
3.3.2 Results and discussion: In Figure 10 three examples of intersection discovery are given. First, dense clusters of stop events are found in the direction of vehicles' movement. Adjacent clusters of similar direction are merged (3i step) as they come from vehicles that move along the same road segments and stop at the same candidate reference spot (in (a) blue and yellow clusters, in (b) purple and green, in (c) purple and blue and yellow and purple). In a third step, representative points (denoted with six-pointed star symbols) of the clusters are determined among members of the clusters. The relative positions of the clusters are analysed finding clusters of conflicting directions (3ii step). These clusters suggest that the traffic participants might stopped because the road they drive on intersects other, so they are grouped in a new cluster which contains the conflicting ones (in (a) the blue and purple, in (b) the green and orange, in (c) purple and blue and yellow and orange). From the new clusters the centres of the intersections can be estimated by finding the points along the direction of their movement where the conflicting clusters intersect.

A weakness of this method is that it returns in some cases false positive reference spots. Such an example is shown in Figure 10 (c), where the orange and yellow clusters suggest an intersection in a location where there is no such. These cases should be further clarified in a post-processing step. Also, another problem is that this method results in many single clusters (not grouped in new ones in the last step), such as the green in (c), suggesting that they should be also further explored. Such a potential junction has to be confirmed, once new measurements occur, which allow to apply the qualitative spatial reasoning rules from Figure 9. Yield-controlled intersections or others where mainly vehicles from one road have to stop could be also inferred by combining the turning-point approach that was discussed earlier in 2.2 with ours.

Currently we extend the proposed method by making use of the "evidence" that turning points provide. Given that this research is motivated by non-rich datasets, the after-stop behaviour (such as turn) may clarify cases like the one discussed last. Also originally this method was made for detecting intersections where no samples from the intersected roads exist in the dataset (Figure 10, (b)), so it makes sense to apply it after having found turning points. It would be interesting also to explore low speed events and not solely relying on zero speed samples. Even in stop controlled junctions drivers often pass them without stopping but by slowing down and quickly checking the other direction roads.

\section{CONCLUSIONS}

In this paper we explored the idea of detecting intersections by analysing stop events under the assumption that they are usually aggregated on junction locations. Clusters of stop events are considered as candidate reference spots that are found in a region-based way, and by resolving the relative directions of the clusters on such spots enables their categorisation as junctions or not. As a next step we will try to eliminate false positive suggestions and the single clusters in no-conflicting context by taking advantage of the after stance behaviour and low speed events. Another issue to be treated in future work is the incremental aggregation of knowledge and confidence in candidate junctions, when new trajectories get available. These candidate junctions can be used as prior knowledge for the reasoning process and thus can speed up the analysis. An important issue is to determine the reliability of a candidate junction. To this end, measures such as number of stops, but also temporal coherence of the stops can be used.
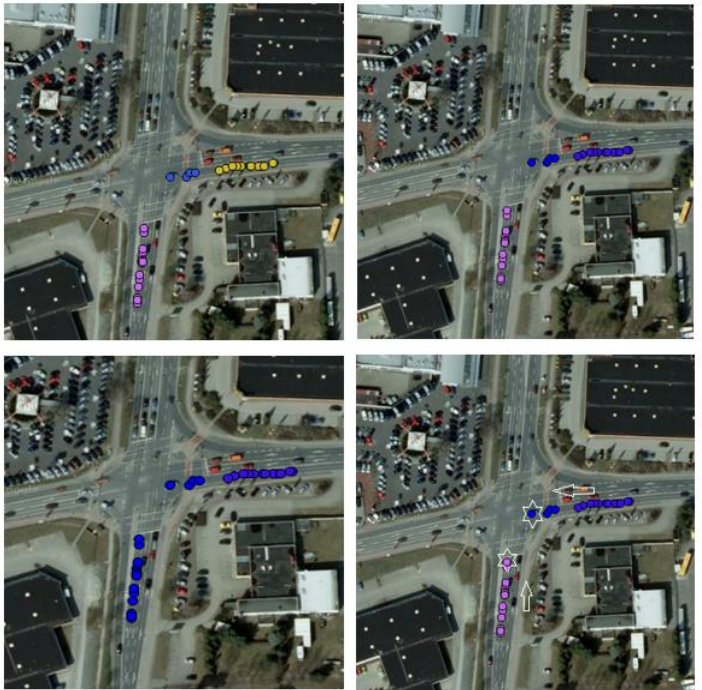

(a)
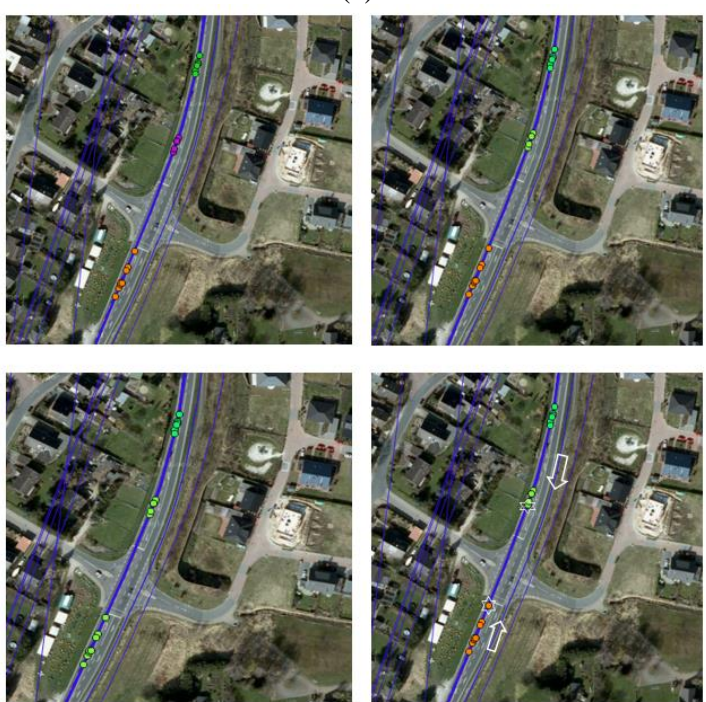

(b)

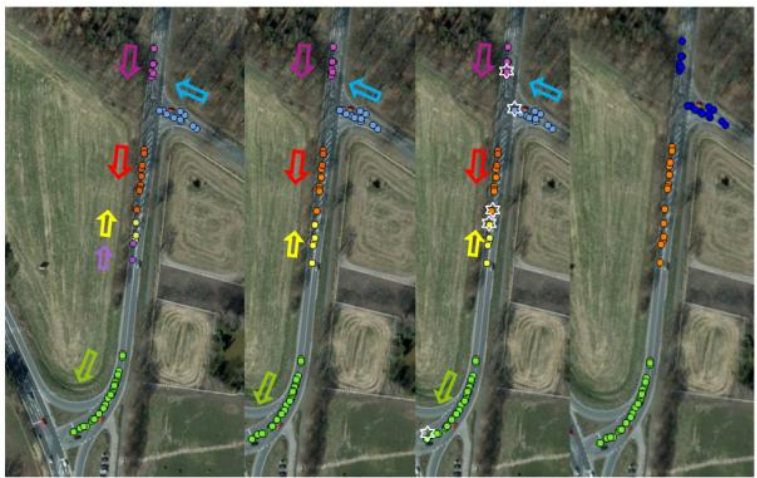

(c)

Figure 10. Clockwise from upper-left (a-b) and from left to right(c): clusters found with DBSCAN, merging of similar direction close clusters and determination of representative points (six-pointed stars), region-based clustering using relational reasoning suggests the existence of an intersection (in (c) two junctions) 


\section{ACKNOWLEDGEMENT}

The research presented in this paper is funded by IAV GmbH. The authors gratefully acknowledge this support.

\section{REFERENCES}

Agamennoni, G., Nieto, J., and Nebot, E., 2009. Mining gps data for extracting significant places. In: IEEE International Conference on Robotics and Automation, ICRA '09, pp. 855862 .

Alvares, L.O., Bogorny, V., Kuijpers, B., Jose Antonio Fernandes de Macedo, Moelans, B., and Vaisman, A., 2007. A model for enriching trajectories with semantic geographical information. In: Proceedings of the 15th annual ACM international symposium on Advances in geographic information systems (GIS '07), New York, NY, USA, pp. 22:122:8.

Biagioni, J., and Eriksson, J., 2012. Map inference in the face of noise and disparity. In: Proceedings of the 20th International Conference on Advances in Geographic Information Systems (SIGSPATIAL '12), New York, NY, USA, pp. 79-88.

Buchin, M., Driemel, A., van Kreveld, M., and Sacristán, V., 2010. An algorithmic framework for segmenting trajectories based on spatio-temporal criteria. In: Proceedings of the 18th SIGSPATIAL International Conference on Advances in Geographic Information Systems (GIS '10), New York, NY, USA, pp. 202-211.

Cao, L., and Krumm, J., 2009. From gps traces to a routable road map. In: Proceedings of the $17^{\text {th }}$ ACM SIGSPATIAL International Conference on Advances in Geographic Information Systems, GIS '09, New York, NY, USA, pp. 3-12.

Chen, Y., and Krumm, J., 2010. Probabilistic modeling of traffic lanes from gps traces. In: Proceedings of the 18th SIGSPATIAL International Conference on Advances in Geographic Information Systems, GIS '10, New York, NY, USA, pp. 81-88.

Chigorin, A., and Konushin, A., 2013. A system for large-scale automatic traffic sign recognition and mapping. In: ISPRS Annals of the Photogrammetry, Remote Sensing and Spatial Information Sciences, Antalya, Turkey, Vol. II-3/W3, CMRT13 - City Models, Roads and Traffic 2013, pp. 13-17.

Eriksson, J., Girod, L., Hull, B., Newton, R., Madden, S., and Balakrishnan, H., 2008. The pothole patrol: using a mobile sensor network for road surface monitoring. In: Proceedings of the 6th international conference on Mobile systems, applications, and services (MobiSys '08), New York, NY, USA, pp. 29-39.

Ester, M., Kriegel, H.-P., Sander, J., and Xu, X., 1996. A Density-Based Algorithm for Discovering Clusters in Large Spatial Databases with Noise. In: Proceedings of the Second International Conference on Knowledge Discovery and Data Mining (KDD-96), Portland, Oregon, pp. 226-231.

Fairfield, N., and Urmson, C., 2011. Traffic light mapping and detection. In: 2011 IEEE International Conference on Robotics and Automation (ICRA), Shanghai, pp. 5421-5426.
Fathi, A., and Krumm, J., 2010. Detecting road intersections from GPS traces. In: Geographic Information Science, Springer Berlin Heidelberg, pp. 56-69.

Hamilton-Baillie, B., and Jones, P., 2005. Improving traffic behaviour and safety through urban design. In: Proceedings of the Institution of Civil Engineers-Civil Engineering, 158(5), pp. 39-47.

Hu, S., Su, L., Liu, H., Wang H., and Abdelzaher, T. F., 2013. Poster abstract: SmartRoad: A crowd-sourced traffic regulator detection and identification system. In: 2013 ACM/IEEE International Conference on Information Processing in Sensor Networks (IPSN), Philadelphia, PA, pp. 331-332.

Karagiorgou, S., and Pfoser, D., 2012. On vehicle tracking databased road network generation. In: Proceedings of the 20th International Conference on Advances in Geographic Information Systems, California, pp. 89-98.

Li, Z., Ding, B., Han, J., Kays, R., and Nye, P., 2010. Mining periodic behaviors for moving objects. In: Proceedings of the 16th ACM SIGKDD international conference on Knowledge discovery and data mining (KDD '10), New York, NY, USA, pp. 1099-1108.

Li, J., Qin, Q., Han, J., Tang, L.-A., and Lei, K. H., 2015. Mining Trajectory Data and Geotagged Data in Social Media for Road Map Inference. Transactions in GIS, 19, pp. 1-18.

Makris, D., and Ellis, T., 2002. Path detection in video surveillance. Image and Vision Computing, 20(12), pp. 895903.

Makris, D., and Ellis, T., 2003. Automatic learning of an activity-based semantic scene model. In: Proceedings of IEEE Conference on Advanced Video and Signal Based Surveillance, pp. 183-188.

Mapscape. http://www.mapscape.eu/telematics/incrementalupdating.html (04 April 2016).

Mathur, S., Jin, T., Kasturirangan, N., Chandrasekaran, J., Xue, W., Gruteser, M., and Trappe, W, 2010. ParkNet: drive-by sensing of road-side parking statistics. In: Sujata Banerjee; Srinivasan Keshav \& Alec Wolman, ed., 'MobiSys', ACM, pp. 123-136.

Mohan, P., Padmanabhan, V. N., and Ramjee, R., 2008. Nericell: rich monitoring of road and traffic conditions using mobile smartphones. In: Proceedings of the 6th ACM conference on Embedded network sensor systems, pp. 323-336.

Niehofer, B., Burda, R., Wietfeld, C., Bauer, F., and Lueert, O., 2009. GPS Community Map Generation for Enhanced Routing Methods Based on Trace-Collection by Mobile Phones. In: Proceedings of the 2009 First International Conference on Advances in Satellite and Space Communications (SPACOMM '09), Washington, DC, USA, pp. 156-161.

Palma, A.T., Bogorny, V., Kuijpers, B., and Alvares, L.O., 2008. A clustering-based approach for discovering interesting places in trajectories. In: Proceedings of the 2008 ACM Symposium on Applied Computing, SAC '08, New York, NY, USA, pp. 863-868. 
Pribe, C., and Rogers, S., 1999. Learning To Associate Observed Driver Behavior with Traffic Controls. Transportation Research Record: Journal of the Transportation Research Board, 1679, pp. 95-100.

Qu, Y., Lee, K., and Lee, I., 2010. Making sense of clusters through qualitative spatial reasoning. 6th International Conference on Advanced Information Management and Service (IMS), Seoul, pp. 67-72.

Schroedl, S., Wagstaff, K., Rogers, S., Langley, P., and Wilson, C., 2004. Mining gps traces for map refinement. Data Mining and Knowledge Discovery, 9(1), pp. 59-87.

Sester, M., Feuerhake, U., Kuntzsch, C., and Zhang, L., 2012. Revealing Underlying Structure and Behaviour from Movement Data, KI - Künstliche Intelligenz, 26(3), pp. 223-231.

Sester, M., Feuerhake, U., Kuntzsch, C., and Zourlidou, S., 2015. Interpretation of Moving Point Trajectories, Photogrammetric Week 15, Stuttgart, Germany.

Wang, Y., Liu, X., Wei, H., Forman, G., Chen, C., and Zhu, Y., 2013. Crowdatlas: Self-updating maps for cloud and personal use. In: Proceeding of the 11th Annual International Conference on Mobile Systems, Applications, and Services, MobiSys '13, New York, NY, USA, pp. 27-40.

Wu, J., Zhu, Y., Ku, T., and Wang, L., 2013. Detecting Road Intersections from Coarse-gained GPS Traces Based on Clustering. Journal of Computers, 8(11), pp. 2959-2965.

Xie, X., Bing-YungWong, K., Aghajan, H., Veelaert, P., Philips, W., 2015. Inferring Directed Road Networks from GPS Traces by Track Alignment. ISPRS International Journal of Geo-Information, 4, pp. 2446-2471.

Zhang, L., and Sester, M., 2010. Incremental Data Acquisition from GPS-Traces. In: Proceedings of ISPRS Commission II, Orlando, USA.

Zhang, L., Thiemann, F., and Sester, M., 2010. Integration of gps traces with road map. In: Proceedings of the Second International Workshop on Computational Transportation Science, IWCTS '10, New York, NY, USA, pp. 17-22.

Zhu, Y., Liu, X., and Wang, Y., 2013. Pervasive urban sensing with large-scale mobile probe vehicles. International Journal of Distributed Sensor Networks, 2013.

Zourlidou, S., and Sester, M., 2015a. Towards regulation-aware navigation: a behavior-based mapping approach. In: 18th AGILE Conference on Geographic Information Science, Lisbon, Portugal.

Zourlidou, S., and Sester, M., 2015b. Road regulation sensing with in-vehicle sensors. In: Proceedings of the 3rd AGILE Phd School, Champs sur Marne, France, 15-17-September 2015, published at http://ceur-ws.org. 